\title{
Loop spin effects in intense background fields
}

\author{
A. Ilderton, ${ }^{*}$ B. King, ${ }^{\dagger}$ and S. Tang $\oplus^{\ddagger}$ \\ Centre for Mathematical Sciences, University of Plymouth, Plymouth, PL4 8AA, United Kingdom
}

(Received 25 August 2020; accepted 30 September 2020; published 21 October 2020)

\begin{abstract}
Radiative and nonradiative electron spin-flip probabilities are analyzed in both plane wave and focused laser backgrounds. We provide a simple and physically transparent description of spin dynamics in plane waves, and demonstrate that there exists a kinematic regime in which the usual leading-order perturbative hierarchy of quantum electrodynamics is reversed, and nonradiative loop effects dominate over radiative tree-level spin flips. We show that while this loop dominance becomes suppressed in focused laser pulses due to a high sensitivity to field geometry, there is nevertheless a regime in which, in principle, loop effects on spin transitions can be discerned.
\end{abstract}

DOI: 10.1103/PhysRevD.102.076013

\section{INTRODUCTION}

Intense laser experiments have begun to probe the nonlinear quantum regime of light-matter interactions $[1,2]$. As such, interest has grown in how to properly account for quantum spin effects in strong background fields, in particular within the framework of extended particle-in-cell (PIC) codes which underlie the analysis of intense laser experiments [3-5].

Radiative spin flip in photon emission from an electron in a laser background through nonlinear Compton scattering $[6,7]$ has been studied in a constant crossed field [8-10] and in pulses [11-15], for which a comprehensive density matrix formalism has also been developed [16]. Using the locally constant field approximation (LCFA) [8], this radiative spin flip has been included in numerical schemes [17-19]. However, the spin can also change in a nonradiative way, through quantum loop effects [20-22]. (See [23-30], also [31-34], for investigations of higher loop orders in strong fields, which has recently seen renewed interest.) It has been suggested to include nonradiative spin changes in numerical codes, using the Bargmann-MichelTelegdi (BMT) equation [35-37]. However, a generalized BMT equation could in principle include both nonradiative and radiative processes [38], as it comes from an expectation value. In order to have a consistent approach which avoids e.g., double-counting, it is important to have a clear

\footnotetext{
*anton.ilderton@plymouth.ac.uk

†b.king@plymouth.ac.uk

*suo.tang@plymouth.ac.uk
}

Published by the American Physical Society under the terms of the Creative Commons Attribution 4.0 International license. Further distribution of this work must maintain attribution to the author(s) and the published article's title, journal citation, and DOI. Funded by SCOAP ${ }^{3}$. understanding of the role of radiative and nonradiative processes in spin dynamics, especially given the known difficulties of closely related topics [39].

In this paper we compare radiative and nonradiative spinflip probabilities in strong background fields. In Sec. II we first make clear that, in the absence of emissions or loop effects, a plane wave background cannot change the spin state of an electron. From this we clarify the interpretation of the leading-order BMT equation in a plane wave background. We then show in Sec. III that, for plane waves, even though the nonradiative loop effect due to spin flip is $O\left(\alpha^{2}\right)$ and spin flip due to radiative effects $O(\alpha)$, there is a kinematic regime where the leading-order perturbative hierarchy is reversed, and the loop effect dominates over tree-level effects.

Various mechanisms to extend our results to realistic focused pulses are discussed in Sec. IV. We identify a new shortcoming of the commonly used locally constant field approximation, and analyze the extent to which the "full" BMT equation can be used to model the nonradiative spin flip. This investigation allows us to consider the spin flip in focused Gaussian pulses in Sec. V. We find that while the loop dominance of the plane wave case does not extend to realistic beams, due to focusing effects breaking the symmetry of a plane wave and allowing spin-flipping to occur at the $O\left(\alpha^{0}\right)$ level, show that despite this, the effect of the loop can still be accessed, in principle, through tail-on collisions of electrons with laser pulses, in contrast to the commonly considered head-on geometry. We conclude in Sec. VI.

\section{A. Notation and conventions}

Initially we model the laser as a plane wave, thus depending on the lightlike direction $n \cdot x$ where $n^{2}=0$. We can always choose $n \cdot x=t-z \equiv x^{-}$, which is a 
natural choice of "lightfront" time direction [40-43]. The remaining directions are $x^{+}=t+z$ and $x^{\perp}=\left(x^{1}, x^{2}\right)$. For momenta we define $p_{ \pm}=\left(p_{0} \pm p_{3}\right) / 2$ and $p_{\perp}=\left(p_{1}, p_{2}\right)$. The plane wave itself is described by the potential $a_{\mu}\left(x^{-}\right)=\delta_{\mu}^{\perp} a_{\perp}\left(x^{-}\right)$in which the two components of $a_{\perp}$ are the $x^{-}$-integrals, starting from $x^{-}=-\infty$ of the wave's two electric field components; this aids the physical interpretation [44].

\section{ELECTRON SPIN IN LASER FIELDS}

Spin in intense laser-matter interactions is commonly considered "classically" [35,36] through the BMT equation [45] which describes the evolution of the spin vector. However, spin is a purely quantum effect, and we will see that the clearest physical understanding of spin in a laser background ultimately comes from considering the quantum theory. In order to maintain contact with both approaches, though, we will here develop the classical and quantum descriptions somewhat in parallel.

\section{A. Lightfront helicity}

An electron has, as well as its momentum $p_{\mu}$, a spin, which can be expressed in terms of a linear combination of two spin states. For particles at rest, these are eigenstates of the spin operator in a chosen direction, with eigenvalues \pm 1 (or "spin up" and "spin down"). There are many possible choices of basis.

The associated covariant spin vector $s^{\mu}$ obeys the two conditions $s \cdot p=0$ and $s \cdot s=-1$. Therefore $s^{\mu}$ has two degrees of freedom, which corresponds to there being two independent spin states. There are many choices of $s^{\mu}$. While all choices agree on what, say, "spin up in the $z$-direction" is for particles at rest, there are ambiguities for particles in motion because there are several inequivalent ways to boost to the same momentum.

It is helpful to choose a particular basis of states and corresponding $s^{\mu}$ which makes calculations simple, and makes the physics manifest both in vacuum and in a plane wave background. The following simple argument motivates our choice. The only vectors available from which we can construct $s^{\mu}$ are $p^{\mu}$ and the propagation direction of the plane wave, $n^{\mu}$. Taking a linear combination of these, one easily finds that

$$
s^{\mu}= \pm \frac{1}{m}\left(p^{\mu}-\frac{m^{2}}{n \cdot p} n^{\mu}\right)
$$

satisfies the two required conditions $s \cdot p=0$ and $s^{2}=-1$. For a particle at rest, these vectors are $s^{\mu} \rightarrow \pm(0,0,0,1)$, which is just spin up or spin down in the $z$-direction.

Writing $\mathrm{p} \equiv\left(p^{-}, p^{\perp}\right)$ for the three lightfront "spatial" components of momentum, we observe that $s \propto p$, similar to the usual (Jacob-Wick) definition of helicity [46], where one would have $s \propto \boldsymbol{p}$ for the Cartesian vector components.
This choice of $s^{\mu}$ is exactly that of "lightfront helicity" used in lightfront field theory [41,42,47], where $n^{\mu}$ arises through the choice of time direction. Lightfront helicity states have the special property that the helicity, call it $\sigma$, is equal to the expectation value of the spin in the $z$-direction, $\sigma= \pm 1$, in all Lorentz frames, so that we may talk of spin and helicity interchangeably. For a thorough discussion see [47].

The two spin states of the electron are represented by two spinors, $u_{p \sigma}$. Define the Pauli-Lubanski (pseudovector) operator [48] by

$$
W_{\mu}:=-\frac{1}{2} \epsilon_{\mu \nu \zeta \rho} P^{\nu} M^{\zeta \rho},
$$

where, in the spinor representation, $M^{\zeta \rho}=(i / 4)\left[\gamma^{\zeta}, \gamma^{\rho}\right]$ and $P \rightarrow p$, the momentum of the state. The corresponding classical spin vectors are just the expectation values of the Pauli-Lubanski operator in the two spin states:

$$
s^{\mu}=-\frac{2}{m} \frac{\left\langle p ; \sigma\left|W^{\mu}\right| p ; \sigma\right\rangle}{\langle p ; \sigma \mid p ; \sigma\rangle} .
$$

We want the states corresponding to lightfront helicity. These are the eigenstates of $W_{\mu}$ contracted with the spin vector ([48], Sec. 2.2). Write $h_{p}^{\mu}$ for the positive sign solution to (1), where subscript $p$ reminds us that the electron has momentum $p_{\mu}$. Defining $L_{p} \equiv 2 h_{p} \cdot \mathrm{W} / \mathrm{m}$, our states obey

$$
L_{p} u_{p \sigma}=\frac{1}{m} \gamma_{5} h_{p} \not p u_{p \sigma}=\sigma u_{p \sigma} .
$$

Their explicit forms are given in Appendix. In terms of the $u$-spinors, (3) becomes

$$
-\frac{1}{m^{2}} \bar{u}_{p \sigma} W^{\mu} u_{p \sigma}= \pm h_{p}^{\mu}
$$

The usefulness of the lightfront helicity basis becomes clear when we turn to the properties of electron spin in an external plane wave. Here the spin structure of electron states, as described by the Volkov solutions [49], changes from $u_{p \sigma}$ to

$$
u_{\pi \sigma}\left(x^{-}\right):=\left(1+\frac{\not h \phi\left(x^{-}\right)}{2 n \cdot p}\right) u_{p \sigma} .
$$

To understand the notation $u_{\pi \sigma}$ recall first that the timedependent kinetic momentum of a particle in a plane wave is

$$
\pi_{\mu}\left(x^{-}\right)=p_{\mu}-a_{\mu}\left(x^{-}\right)+n_{\mu} \frac{2 a\left(x^{-}\right) \cdot p-a^{2}\left(x^{-}\right)}{2 n \cdot p},
$$

and that, by construction, $\not t u_{\pi \sigma}=m u_{\pi \sigma}$ in order for the Dirac equation to be obeyed. In other words, $u_{\pi \sigma}$ is the $u$-spinor for on-shell momentum $\pi$. Now consider the helicity. A direct 
calculation shows that the lightfront helicity operator commutes with the additional spin structure in (6):

$$
L_{\pi} u_{\pi \sigma}=\left(1+\frac{\not h \phi\left(x^{-}\right)}{2 n \cdot p}\right) L_{p} u_{p \sigma}=\sigma u_{\pi \sigma} .
$$

This means that the helicity eigenstates remain eigenstates in the background, even though the momentum of the state changes in time. As this is a basis, we see that the quantum spin state of an electron cannot be changed by propagation through a plane wave background alone.

\section{B. The BMT equation}

In light of this result, we turn to the leading order (in $\alpha$ ) BMT equation. Observe that even though the state of the electron remains unchanged in a plane wave, the associated spin vector does change [50], from $h_{p}$ to $h_{\pi}$. Using (7) we can express $h_{\pi}$ in terms of $h_{p}$ as

$$
\begin{aligned}
h_{\pi}^{\mu} & \equiv \frac{1}{m} \pi^{\mu}-\frac{m}{n \cdot \pi} n^{\mu} \\
& =h_{p}^{\mu}-\frac{n \cdot h_{p}}{n \cdot p} a^{\mu}+\left(\frac{a \cdot h_{p}}{n \cdot p}-\frac{a^{2} n \cdot h_{p}}{2 n \cdot p^{2}}\right) n^{\mu},
\end{aligned}
$$

in which we have used $a \cdot h_{p}=a \cdot p / m$ and $n \cdot h_{p}=$ $n \cdot p / m$. Written as in (9), we recognize $h_{\pi}$ as nothing but the solution to the leading-order BMT equation in a plane wave,

$$
\frac{\mathrm{d} h_{\mu}}{\mathrm{d} x^{-}}=\frac{1}{n \cdot p} F_{\mu \nu} h^{\nu}
$$

with initial condition $h^{\mu}=h_{p}^{\mu}$, where $F_{\mu \nu}=\partial_{\mu} a_{\nu}-\partial_{\nu} a_{\mu}$ is the electromagnetic field strength tensor. (We have written the equation in terms of lightfront time rather than proper time, as is more common in the literature.) The interpretation of the BMT equation now becomes clear: the classical spin vector precesses, but only to account for the fact that the electron momentum is changing, and this is captured by the leading-order BMT equation. It does not describe a change in the spin state of the electron, though, because there is no change. This is shown by the quantum calculations above, and will be recovered from quantum electrodynamics (QED) scattering calculations below.

\section{Summary}

The physics of spin in plane wave backgrounds is made transparent by our calculation: the background changes the momentum of an electron, but not its spin state. We have shown this essential result using a natural lightfront helicity basis, but it can be proven in any basis: for any $s_{\pi}^{\mu}$ obeying the lowest order BMT equation, there is a corresponding $L_{\pi}$ obeying (8). Lightfront helicity has the advantages of allowing simple, explicit calculations, including, note, an essentially algebraic solution to the leading-order BMT differential equation, above.

It follows that if one wants to change the spin state of an electron in a plane wave, then emissions or loop corrections are required. This is the topic of the next section, in which we will also see that lightfront helicity states have interesting properties.

\section{SPIN-FLIP IN PLANE WAVE BACKGROUNDS}

The spin state of an electron can be changed in plane wave backgrounds via both radiative and nonradiative processes. Here we describe contributions to each. It is instructive to organize the presentation in powers of the fine structure constant $\alpha$.

Working in the lightfront helicity basis above, the probability of helicity/spin flip from a state $\sigma$ to a state $-\sigma$ in any given process, using $S$-matrix methods and Volkov wavefunctions [49], is well documented [8-10,16, $20,51,52]$, so we present only final results here.

\section{A. Order $\boldsymbol{\alpha}^{\mathbf{0}}$ : Propagation}

To zeroth order in $\alpha$, the only contribution to spin flip can be nonradiative, see the left hand diagram in Fig. 1. The corresponding scattering amplitude comes from the double LSZ amputation of the Volkov propagator and has the structure [53]

$$
S_{f i} \propto \bar{u}_{p \sigma^{\prime}} h u_{p \sigma} \propto \delta_{\sigma \sigma^{\prime}}
$$

so that there can be no spin flip. This confirms the result encountered above, that a particle's quantum spin state cannot be changed by propagation through a plane wave alone (i.e., propagation in the absence of loops or emissions). We underline that it is the contribution from this amplitude which leads to the leading-order BMT
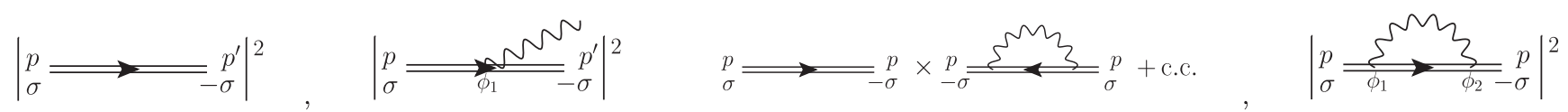

FIG. 1. Diagrams contributing to spin flip of an electron, initial momentum $p$ and spin $\sigma$. Left: nonradiative spin flip at zeroth order in $\alpha$, from propagation through the field. Middle two diagrams: both radiative and nonradiative spin flip receive contributions at order $\alpha$. Right: nonradiative spin flip at order $\alpha^{2}$, from the one-loop propagator correction. Double lines denote background-dressed propagators; diagrams should be understood as LSZ-amputated scattering amplitudes. 
equation (10), which can then only describe the precession of the classical spin vector, not a change in spin state.

\section{B. Order $\alpha$ : Radiative spin flip}

The lowest order contribution to radiative spin flip comes at order $\alpha$, from nonlinear Compton scattering (NLC) at tree level. To present the probability, we define for convenience $\varphi$ and $\vartheta$ as two lightfront times scaled by some typical (central) frequency scale $\omega$ of the plane wave background. The parameter $\eta:=\omega n \cdot p / m^{2}$ then characterizes the invariant energy of the interaction. Define the floating average $\langle f\rangle=\frac{1}{\vartheta} \int_{\varphi-\vartheta / 2}^{\varphi+\vartheta / 2} d \phi f(\phi)$ for any $f$, and in terms of this the normalized Kibble mass $\Lambda=1-\left\langle a^{2}\right\rangle / m^{2}+\langle a\rangle^{2} / m^{2}$. The probability of (radiative) spin flip in NLC may then be written

$$
\mathrm{P}_{\mathrm{NLC}}=\frac{\alpha}{2 \pi \eta} \int \mathrm{d} \varphi \int_{0}^{\infty} \mathrm{d} \vartheta \frac{\partial \ln \Lambda}{\partial \vartheta} \mathrm{C}\left(\frac{\vartheta \Lambda}{2 \eta}\right)
$$

in which the function $\mathrm{C}$ arises as an integral over the lightfront momentum fraction $s$ of the emitted photon, $s:=n \cdot \ell / n \cdot p$ for photon momentum $\ell$ :

$$
\mathrm{C}(\mu)=\int_{0}^{1} \frac{\mathrm{d} s s^{2}}{1-s} \sin \left(\frac{\mu s}{1-s}\right) .
$$

Note that $\mathrm{P}_{\mathrm{NLC}}$ is independent of the sign of $\sigma$.

\section{Order $\alpha$ : Interference}

In general, nonradiative spin flip has an order $\alpha$ contribution. This is a quantum interference term, coming from the product of the tree-level propagation diagram and the one-loop propagation diagram, see the third diagram in Fig. 1. However, since the order $\alpha^{0}$ amplitude is diagonal in spin, in a plane wave, the order $\alpha$ contribution is forced to vanish. This result has several important consequences, see below.

\section{Order $\alpha^{2}$ : Nonradiative spin flip}

Due to the vanishing of the propagation (order $\alpha^{0}$ ) and interference (order $\alpha$ ) terms above, nonradiative spin flip receives its leading-order contribution at order $\alpha^{2}$. This comes from the one-loop correction to the propagator $[20,21,27,28]$, mod squared, see the right hand diagram in Fig. 1. With the same notation as used above for nonlinear Compton scattering, the leading-order nonradiative spin-flip probability may be written

$$
\mathrm{P}_{\text {loop }}=\frac{\alpha^{2}}{(2 \pi \eta)^{2}}|\mathrm{M}|^{2}
$$

where, for $\Delta a_{\mu}=a_{\mu}(\varphi+\vartheta / 2)-a_{\mu}(\varphi-\vartheta / 2)$,

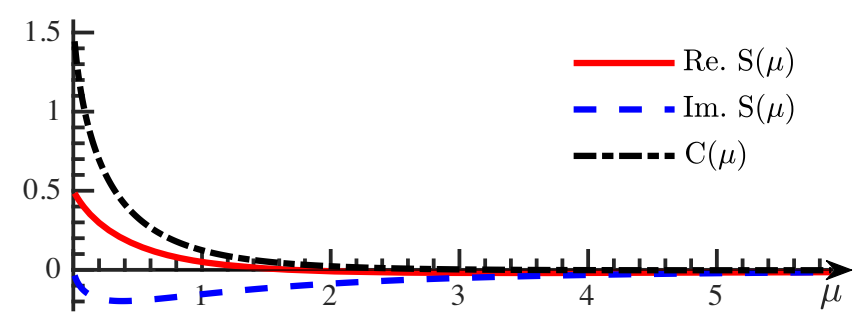

FIG. 2. Plots of $\mathrm{C}(\mu)$ in (13) and $\mathrm{S}(\mu)$ in (16) showing that these functions do not give significant contributions for large arguments.

$$
\mathrm{M}=\int \mathrm{d} \varphi \int_{0}^{\infty} \frac{\mathrm{d} \vartheta}{\vartheta}\left(\frac{\Delta a_{2}}{2 m}+i \sigma \frac{\Delta a_{1}}{2 m}\right) \mathrm{S}\left(\frac{\vartheta \Lambda}{2 \eta}\right)
$$

in which ${ }^{1} \mathrm{~S}(\mu)$ arises as an integral over the lightfront momentum fraction of the intermediate virtual photon,

$$
\mathrm{S}(\mu)=\int_{0}^{1} \mathrm{~d} s s \mathrm{e}^{-i \mu \frac{s}{1-s}}
$$

With expressions (12) and (14) in hand we can begin to discuss the physics of the spin-flip probabilities. We first note that the radiative flip probability (12) is independent of the sign of $a_{\mu}$. The nonradiative flip probability, though, is strongly dependent on the sign of $a_{\mu}$ : because the real/ imaginary parts of $S$ do not change sign (see Fig. 2), the sign of the integrand in (15) is determined by the sign of $a_{\mu}$. It follows that there can be no nonradiative spin flip if the potential is an even function, because then the integral over $\varphi$ in (15) gives zero.

This difference in dependence on the driving laser field appears because we look at flips between lightfront helicity states. For electron spins polarized in other directions, the radiative probability can show the same sign dependence as the nonradiative probability, see [16].

\section{E. Order $\alpha^{2}$ : Radiative}

Radiative spin flip has two order $\alpha^{2}$ contributions. The first comes from double nonlinear Compton scattering (two photon emission) at tree level, mod squared. The second is an interference contribution from the cross term of (single) nonlinear Compton at tree level and at one loop. However, the probability for one spin flip to occur in double nonlinear Compton scattering scales as $\alpha^{2} \eta^{2} \Phi$, where $\Phi$ is the pulse phase duration. We will see that the most interesting region for the loop is when $\eta<\Phi^{-1}$ and when the pulse is short,

\footnotetext{
${ }^{1}$ We comment that, in the loop calculation, $\varphi$ and $\vartheta$ arise as the average and difference of the lightfront times corresponding to "emission" and "absorption" of the virtual photon, $\vartheta=\phi_{2}-\phi_{1}$ and $\varphi=\left(\phi_{2}+\phi_{1}\right) / 2$ referring to Fig. 1 . In the calculation of the NLC probability, if the real photon is emitted at time $\phi_{1}$ in the scattering amplitude, then $\phi_{2}$ would be the emission time in the conjugate amplitude.
} 
hence we can ignore order $\alpha^{2}$ radiative contributions in the regime of interest.

\section{F. Comparison}

The different field dependencies of the spin-flip probabilities found above prompts us to ask when one of the processes, radiative or nonradiative, can dominate over the other. To this end, consider the behavior of the probabilities for small $\eta$, beginning with the nonradiative result (14), which is exact to order $\alpha^{2}$. The main contribution to the integrand in (15) originates in the region where the argument of $\mathrm{S}$ is small, see Fig. 2. Thus, if $\eta \rightarrow 0$, the main contribution comes from $\vartheta \ll 1$. This allows us to approximate the integral, in the small- $\eta$ limit, using a small- $\vartheta$ expansion, setting $\Lambda \approx 1-\vartheta^{2} a^{2} / 12 \approx 1, \vartheta \Lambda / 2 \eta \approx \vartheta / 2 \eta$, and $\Delta a \approx m \vartheta(0, \boldsymbol{\varepsilon})$, where $\boldsymbol{\varepsilon}=\boldsymbol{\varepsilon}(\varphi):=\mathbf{a}^{\prime}(\varphi) / m$ is the normalized electric field. Equation (15) can then be integrated analytically to give

$$
\begin{aligned}
\mathrm{M} & \approx \eta \int \mathrm{d} \varphi \int_{0}^{\infty} \mathrm{d} \mu \mathrm{S}(\mu)\left(\boldsymbol{\varepsilon}_{2}+i \sigma \boldsymbol{\varepsilon}_{1}\right) \\
& =-\frac{i \eta}{2 m}\left[a_{2}(\infty)+i \sigma a_{1}(\infty)\right] .
\end{aligned}
$$

Inserting into (14), the nonradiative spin-flip probability behaves in the limit $\eta \rightarrow 0$ as

$$
\mathrm{P}_{\text {loop }} \approx \frac{\alpha^{2}}{(2 \pi)^{2}}\left|\frac{a_{\perp}(\infty)}{2 m}\right|^{2},
$$

which is independent of $\eta$. This behavior holds for pulses with a unipolar structure [44,50], $a_{\perp}(\infty) \neq 0$ which essentially means that although the fields oscillate, they are "more" positive than negative (or vice versa). Such pulses exhibit the electromagnetic memory effect [44,54]. For "whole-cycle" pulses, $a_{\perp}(\infty)=0$ there is no memory effect, and the leading-order contribution at small $\eta$ is suppressed as a positive power of $\eta$.

The corresponding low- $\eta$ behavior of the NLC probability is found similarly; it scales quadratically with $\eta$ as

$$
\mathrm{P}_{\mathrm{NLC}} \approx \frac{35 \alpha}{48 \sqrt{3}} \eta^{2} \int \mathrm{d} \varphi|\boldsymbol{\varepsilon}(\varphi)|^{3} .
$$

Comparing (18) and (19) suggests that at small $\eta$ there can be a reversal of the usual hierarchy of QED perturbation theory, with the one-loop, order $\alpha^{2}$ nonradiative probability dominating over the tree-level, order $\alpha$, radiative probability.

To demonstrate this result, we consider a head-on collision between an electron and a simple model of a unipolar pulse, being a single half-cycle with electric field $\mathbf{E}=E_{0}\left(\cos \omega x^{-}, 0,0\right)$, with $\left|\omega x^{-}\right|<\pi / 2$, meaning normalized potential $a\left(x^{-}\right)=m \xi\left(0,1+\sin \omega x^{-}, 0,0\right)$, where $\xi:=e E_{0} /(m \omega)$ is the dimensionless peak value of $a$. We set $\omega=1.18 \mathrm{eV}(\lambda=1054 \mathrm{~nm})$, for an optical laser and vary
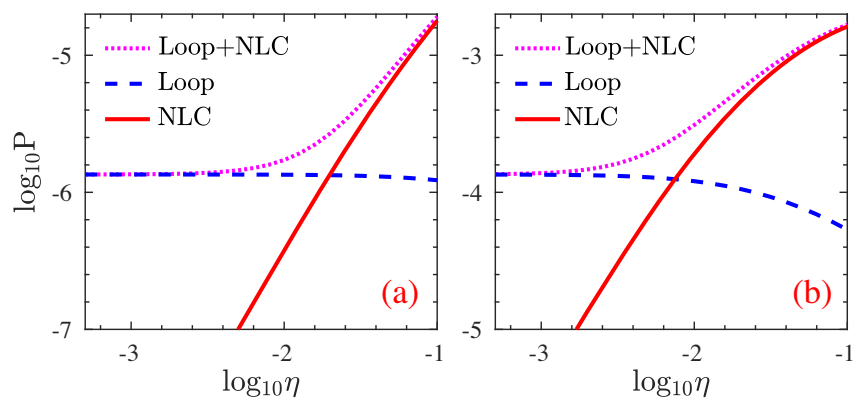

FIG. 3. Radiative and nonradiative spin-flip probabilities (12) and (14), respectively, in the head-on collision of an electron with the half-cycle pulse in the text, as a function of varying electron energy from $55 \mathrm{MeV}\left(\eta=10^{-3.3}\right)$ to $11 \mathrm{GeV}(\eta=0.1)$. Intensities $\xi=1$ (a) and $\xi=10$ (b). The sum of the two probabilities is also shown. The low $\eta$ behaviors (18) and (19) are clearly seen.

the electron energy from $55 \mathrm{MeV}$ to $110 \mathrm{GeV}$, corresponding to $\eta$-parameters from $10^{-3.3}$ up to 1 . We plot the exact probabilities (12) and (14) in Fig. 3.

We see that for smaller $\eta$, the one-loop nonradiative spinflip probability is indeed independent of $\eta$, and orders of magnitude larger than the tree-level radiative probability. The latter increases quickly with $\eta$, confirming the behavior in (19). The crossover point below which the loop begins to dominate lies around $\eta \approx 0.21 \sqrt{\alpha / \xi}$, in which the parameter dependence follows directly from comparing (18) and (19). This corresponds to an approximate electron energy of $0.11\left(\mathrm{~m}^{2} / \omega\right) \sqrt{\alpha / \xi}$ for head-on collisions. The limits (18) and (19) together with the results in Fig. 3 confirm that loop effects can in principle dominate over tree level in electron spin dynamics, when the pulse has a unipolar structure.

\section{THE LCFA AND THE BMT EQUATION}

The question we would like to address is whether the loop dominance found above can in principle be observed in realistic electron-laser collisions. The difficulty is that the radiative and nonradiative spin-flip probabilities for realistic, strong fields are not known. The usual approach to sidestepping this problem is to use a "locally constant field approximation" (LCFA) which, applied to the plane wave probabilities, yields approximate expressions which can be extended to more general fields [8] and implemented in numerical simulations [5]. Regarding spin flip, we will identify here some problems with this method, but also present a resolution.

\section{A. Benchmarking}

The standard method to deriving the LCFA from a given plane wave expression is to perform the same small- $\vartheta$ expansion introduced above (17), but now for arbitrary $\eta$ $[55,56]$. This again allows us to integrate over $\vartheta$. The spinflip probabilities become 

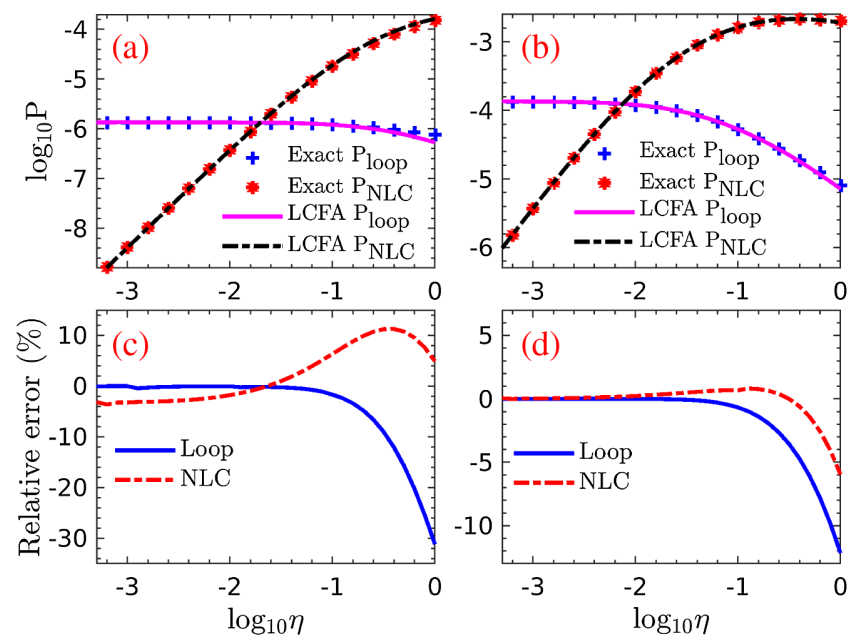

FIG. 4. Benchmarking the LCFA of the radiative and nonradiative spin-flip probabilities against their exact results (12) and (14). The same parameters as in Fig. 3 are used. Left column: $\xi=1$; right column: $\xi=10$. The upper panels (a) and (b) show the LCFA predictions (20) along with the exact results (12) and (14). The lower panels (c) and (d) show the relative error between the LCFA and exact results, $\left(P^{\text {lcfa }}-P^{\text {exact }}\right) / P^{\text {exact }}$.

$\mathrm{P}_{\mathrm{NLC}}=\frac{\alpha}{2 \eta} \int \mathrm{d} \varphi \int_{0}^{1} \mathrm{~d} s \frac{s^{2}}{1-s} \mathrm{Ai}_{1}(z)$,

$\mathrm{P}_{\text {loop }}=\left|\frac{\alpha}{2 \eta} \int \mathrm{d} \varphi \int_{0}^{1} \frac{s \mathrm{~d} s}{\sqrt{z}}[\operatorname{Ai}(z)-i \operatorname{Gi}(z)] \frac{\varepsilon_{2}+i \sigma \varepsilon_{1}}{|\boldsymbol{\varepsilon}|}\right|^{2}$,

in which Ai and Gi are the Airy and Scorer functions [57] with argument $z=\left[s / \chi_{p}(1-s)\right]^{2 / 3}, \chi_{p}=\eta|\boldsymbol{\varepsilon}(\varphi)|$, and $\operatorname{Ai}_{1}(z)=\int_{z}^{\infty} \mathrm{d} x \operatorname{Ai}(x)$.

The first thing to check is that these approximations are capable of accurately reproducing general plane wave results in the regime of interest. Hence we benchmark the LCFA (20) against exact results in Fig. 4: they match very well, in particular at small $\eta$ (which justifies the approximation used to find the small $\eta$ limits). In panels (c) and (d) we plot the relative error between the LCFA and exact results; as $\eta$ increases, the relative error becomes larger, and as the intensity $\xi$ increases, the relative error becomes smaller, as expected of the LCFA. We note that the LCFA provides a good approximation even at $\xi=1$.

\section{B. Spin-flip beyond plane waves and BMT}

In general backgrounds, we expect both order $\alpha^{0}$ (propagation) and order $\alpha^{1}$ (interference) contributions to nonradiative spin flip. If these contributions are to be approximated using the LCFA applied to plane wave results (or inferred from the constant crossed field result) then they are automatically set to zero. This is another shortcoming of the LCFA, see also [27,28,58-60]. As such, we need another method to investigate nonradiative spin flip beyond plane waves. One option is to use the full BMT equation for the spin vector $s^{\mu}$, but there are subtleties to be confronted in doing so, which we address here.

Let $|\psi\rangle$ be a superposition of single electron lightfront helicity states

$$
|\psi\rangle=c_{+}|\pi ;+\rangle+c_{-}|\pi ;-\rangle,
$$

in which $\left|c_{+}\right|^{2}+\left|c_{-}\right|^{2}=1$ and $\pi$ is the instantaneous (timedependent) momentum of the state. Using the definition (3) we write the corresponding classical spin vector as

$$
s^{\mu}=\frac{-2}{m}\left\langle\psi\left|W^{\mu}\right| \psi\right\rangle=\sum_{\sigma^{\prime}, \sigma} c_{\sigma}^{*} c_{\sigma^{\prime}} S_{\sigma \sigma^{\prime}}^{\mu},
$$

in which the "basis elements" $S_{\sigma \sigma^{\prime}}^{\mu}$ are given by $S_{\sigma \sigma^{\prime}}^{\mu}:=-2 / m\left\langle\pi ; \sigma\left|W^{\mu}\right| \pi ; \sigma^{\prime}\right\rangle$ : for explicit expressions see Appendix. They obey the relations

$$
\begin{aligned}
S_{\sigma \sigma^{\prime}}^{2} & =-\delta_{\sigma \sigma^{\prime}}, \quad S_{-+} \cdot S_{+-}=-2, \\
S_{++} \cdot S_{+-} & =0, \quad S_{++} \cdot S_{-+}=0,
\end{aligned}
$$

using which it is easily checked that the probability of observing spin up or spin down in the state (21) can be extracted from the classical spin vector by projecting onto $S_{++}$:

$$
\left|c_{ \pm}\right|^{2}=\left(1 \mp s \cdot S_{++}\right) / 2 .
$$

Observe that this result holds in an arbitrary background if we take $\pi$ to be the instantaneous momentum in that background, because the lightfront helicity basis is background-independent. As such [noting that the ansatz (21) explicitly neglects emissions] we have a method for extracting the nonradiative spin flip probability, in general fields, from the classical spin vector $s^{\mu}$. This can in turn be calculated using the BMT equation.

Given, then, that we want to use the BMT equation to consider spin beyond plane wave backgrounds, it is worth briefly emphasizing what physics the BMT equation does and does not describe. We will continue to focus on plane waves to explore this.

The BMT equation as usually used to describe the (nonradiative) evolution of the spin vector is $[20,38,45]$

$$
\frac{\mathrm{d} s^{\mu}}{\mathrm{d} \tau}=\frac{\mu_{b}+1}{m} F^{\mu \nu} s_{\nu}+\frac{\mu_{b}}{m^{3}} \pi^{\mu}\left(s_{\alpha} F^{\alpha \beta} \pi_{\beta}\right),
$$

in which the anomalous magnetic moment $\mu_{b}$ is taken to be $[20,35,36,38]$

$$
\mu_{b}=\frac{g-2}{2}=\frac{\alpha}{\chi_{p}} \int_{0}^{1} \frac{s \mathrm{~d} s}{\sqrt{z}} \operatorname{Gi}(z),
$$

where $g$ is the electron's gyromagnetic ratio or "g factor". Compare this to the exact evolution equation for (22), which is 


$$
\begin{aligned}
\frac{\mathrm{d} s^{\mu}}{\mathrm{d} x^{-}} & =\sum_{\sigma^{\prime}, \sigma} c_{\sigma}^{*} c_{\sigma^{\prime}} \frac{\mathrm{d} S_{\sigma \sigma^{\prime}}^{\mu}}{\mathrm{d} x^{-}}+\sum_{\sigma^{\prime}, \sigma} S_{\sigma \sigma^{\prime}}^{\mu} \frac{\mathrm{d} c_{\sigma}^{*} c_{\sigma^{\prime}}}{\mathrm{d} x^{-}} \\
& =\frac{1}{n \cdot p} F^{\mu \nu} s_{\nu}+\sum_{\sigma^{\prime}, \sigma} S_{\sigma \sigma^{\prime}}^{\mu} \frac{\mathrm{d} c_{\sigma}^{*} c_{\sigma^{\prime}}}{\mathrm{d} x^{-}},
\end{aligned}
$$

where, in the first term, we have used the result (9). Using that $\mathrm{d} x^{-} / \mathrm{d} \tau=p^{-} / m$ in plane waves, we equate (24) and (26), then project onto $S_{++}^{\mu}$ or $S_{+-}^{\mu}$ in order to obtain the evolution equations

$$
\begin{aligned}
& \frac{\mathrm{d}\left|c_{-}\right|^{2}}{\mathrm{~d} \varphi}=-\frac{\mu_{b}}{2}\left[c_{-} c_{+}^{*}\left(\varepsilon_{x}+i \varepsilon_{y}\right)+c_{+} c_{-}^{*}\left(\varepsilon_{x}-i \varepsilon_{y}\right)\right], \\
& \frac{\mathrm{d} c_{-} c_{+}^{*}}{\mathrm{~d} \varphi}=-\frac{\mu_{b}}{2}\left(1-2\left|c_{-}\right|^{2}\right)\left(\varepsilon_{x}-i \varepsilon_{y}\right),
\end{aligned}
$$

where $\varphi=\omega x^{-}$. For an initial helicity state $|+\rangle$at $\varphi=-\infty$ we have $c_{+}=1$ and $c_{-}=0$. Assuming in the subsequent evolution that $c_{+} \sim 1$ and $c_{-} \sim \alpha$ allows us to approximate the evolution equations as

$$
\frac{\mathrm{d} c_{-}}{\mathrm{d} \varphi}=-\frac{\mu_{b}}{2}\left(\varepsilon_{x}-i \varepsilon_{y}\right)
$$

and so the spin-flip probability as inferred from BMT becomes

$$
\left|c_{-}\right|^{2}=\left|\frac{1}{2} \int \mathrm{d} \varphi\left(\varepsilon_{x}-i \varepsilon_{y}\right) \mu_{b}\right|^{2}
$$

We would expect this to match be the nonradiative spin-flip probability in a plane wave, which we have already seen is well approximated by Eq. (20). We see that this matches (29) exactly, provided that the Airy function contribution from the loop is neglected. This is negligible, compared to the Scorer function, at small $\chi_{p}$. Hence, the BMT equation as applied in the literature uses only part of the full loop expression (inferred from the LCFA); it holds only at low $\chi$, which at fixed intensity corresponds to low energy - this is where the BMT equation can be applied, and this is sufficient for our purposes. Note that in the same limit radiative spin transitions (from photon emission) are suppressed, which is why they can also be dropped from BMT.

\section{LOOP DOMINANCE IN FOCUSED PULSES}

In the above plane wave calculations, a unipolar pulse structure is required to counter the cancellation of oppositesign contributions in (15), coming from oscillations of the field. What we will now show is that it is possible to realize an effective unipolar structure in the collision of electrons with focused laser fields, which in principle allows experimental access to the loop; we will however see that loop effects are more subtle in focused pulses than in plane waves.



FIG. 5. (a) A near tail-on collision between a high-energy electron, momentum $p$, and a focused laser pulse propagating in the $z$-direction, linearly polarized in the $x$-direction. $\lambda$ is the laser wavelength. $\theta$ is the incident angle of the electron in the $x-z$ plane. The electron goes through the pulse center $(0,0,0)$ at $t=0$. The nonzero components of the electromagnetic fields along the electron trajectory are shown in (b) $E_{x}$, (c) $B_{y}$, and (d) $E_{z}$, in ratio to the peak field amplitude $\xi$ : laser focal radius $w=2 \lambda$ and electron incident angle $\theta=10^{\circ}$.

The key is to consider, in contrast to the de-facto setup of intense laser-matter interactions, an almost tail-on collision between the electron and laser. In this geometry a highenergy electron can almost "keep up" with the pulse such that it sees, as it traverses the field, an effectively unipolar, or even same-sign, pulse. We make this concrete in Fig. 5, which shows the effective fields seen by a high-energy electron on a ballistic trajectory crossing a tightly focused Gaussian pulse. The pulse has focal radius $w$, is linearly polarized in the $x$-direction, propagates in the $z$-direction, and has Gaussian temporal profile $\exp \left(-t^{2} / \tau^{2}\right)$ where $\tau=$ $5 T$ for laser period $T$. (See [61] for explicit expressions for the fields.)

Furthermore, in near tail-on collisions $\eta$ is naturally small, $\eta=(1-\beta \cos \theta) \gamma \omega / m$ where $\theta$ is the small angular deviation from fully tail-on. ${ }^{2}$ Therefore this interaction setup is that which could allow the dominance of nonradiative loop effects to be seen.

In Fig. 6, we plot the radiative and nonradiative spin-flip probabilities in near tail-on collisions between a highenergy electron and the Gaussian pulse. The radiative probability is calculated using the LCFA for nonlinear Compton scattering, evaluated along the trajectory of the electron as it traverses the field; this in turn is found by numerically solving the Lorentz force equation. The nonradiative flip probability is calculated by numerically solving the BMT equation (24)-(25), and extracting from the solution the spin flip probability as in (23). We present also the nonradiative probability obtained from entirely neglecting the loop, and setting $g=2$ in the BMT equation.

\footnotetext{
${ }^{2}$ This is provided the electron energy is not too large: this is not a strong constraint as the laser frequency is so low.
} 



FIG. 6. Spin-flip probabilities in tail-on collisions with focused laser pulses, as a function of initial electron energy, $w=2 \lambda$ and $\theta=10^{\circ}$. Laser intensity (a) $\xi=10$ and (b) $\xi=50$. The electron energy changes from $100 \mathrm{MeV}$ to $10 \mathrm{GeV}$. The corresponding change in $\eta$, from $10^{-5.2}$ to $10^{-3.2}$, is shown on the top axis.

The results do not show the same loop dominance as found in plane waves and as shown in Figs. 3 and 4. While nonradiative spin flip continues to dominate at low energies, this comes from the leading-order BMT equation in which loop effects are turned off. Our results do show, though, that loop effects are in principle detectable: there is an energy regime, below the region where radiative spin flip contributes, in which the nonradiative spin flip probability is enhanced by the loop by roughly an order of magnitude.

The reason for the difference in behavior of the spin-flip probabilities in Gaussian beams and plane waves is due to their different field structures, in particular focusing effects. The symmetry of the plane wave background means that the helicity of the electron cannot be changed at the propagator level, and leading-order spin-flip terms are $O\left(\alpha^{2}\right)$. As soon as this symmetry is broken by focusing, terms of $O\left(\alpha^{0}\right)$ and $O\left(\alpha^{1}\right)$ can contribute to the spin flip. This breaking of the plane-wave symmetry can be quantified in a focused Gaussian pulse by the focal expansion parameter $\epsilon:=\lambda / \pi w$ [61]. In the small- $\eta$ regime, the leading-order hierarchy of effects contributing to a spin change in a focused Gaussian pulse is then:

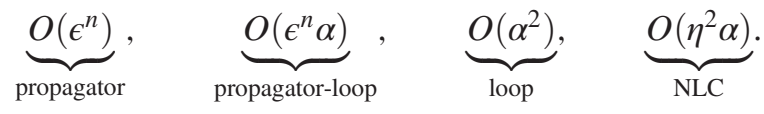

For our parameters, as used in Fig. 6, we have $\epsilon^{5} \approx 10^{-4}>\alpha^{2}$ (while higher orders in $\epsilon$ are smaller than $\alpha^{2}$ ), so to be consistent in our modeling of the Gaussian pulse, we must use expressions for the field which are correct up to fifth order in the focal expansion parameter. Indeed, in the parameter regime presented in Fig. 6, numerical analysis using the lower-order beam model suggest that the loop dominance of the plane-wave case persists, but this is misleading, as large differences appear when order $\epsilon^{3}$ terms are added to the beam, and the loop dominance is mostly lost. However we found that very little change occurred when $\epsilon^{5}$ terms were added. This is consistent with the order-of-magnitude estimates above. If we had instead used the LCFA approximation (20) and neglected the contribution of lower-order effects from focusing, as is the usual way of applying the LCFA, then only the order $\alpha^{2}$ spin-flip contribution would have been included. This would have suggested that also in a focused background, the loop dominance survives, but this would have been incorrect. Thus interference between focusing effects and plane-wave loop effects is crucial to spin flip at low energy in a laser pulse. We conclude from this that spin-effects described by the BMT equation, in a nonplane-wave background, are very sensitive to the structure of that background. The sensitivity is much higher than can currently be controlled in experiment. ${ }^{3}$

One could also consider electron spin dynamics in two counter-propagating pulses. In this setup, however, the electron would itself typically be counter-propagating with one of the pulses, which would lead to a large $\eta$ and hence dominance of radiative spin flip. One might instead consider a traverse collision where an electron propagates perpendicular to the wave vectors in the standing wave at a magnetic node. However, at small $\eta$, the magnetic node solution is quite different from a plane wave, and the leading-order BMT contribution will not be zero, and for this setup, the plane-wave limit is only reached at large $\eta$ [62], which means that again, radiative spin flip would dominate.

\section{CONCLUSIONS}

We have analyzed radiative and nonradiative electron spin flip in intense fields. In the case of plane wave backgrounds, commonly used as a prototype model of intense laser fields, we have found that there exists a kinematic regime in which the usual leading-order perturbative hierarchy of QED is reversed, and where the one-loop nonradiative process dominates over the radiative tree-level process.

This is possible due to two conspiring effects: first, the suppression at low energy (invariant $\eta$ ) of spin flip due to radiative emissions; second, the exact vanishing of the a priori dominant amplitude for spin flip through propagation (without emission of loop effects) at tree level.

While this loop dominance does not extend to more realistic (e.g., Gaussian beam) models of focused laser

\footnotetext{
${ }^{3}$ We briefly mention higher-order nonradiative effects. These can be considered using e.g., the Schwinger-Dyson equations [21]. In plane waves, this introduces an additional spin structure, with which the helicity operator does not commute-this is consistent with our results, since loops can change the spin. Such results are still tied to plane waves and hence miss the leading and next-to-leading-order terms that are nonzero in a focused pulse.
} 
pulses, the influence of the loop on nonradiative spin flip can in principle be accessed experimentally through (near) tail-on collisions between high-energy electrons and tightly focused laser pulses. This geometry, in contrast to the usual setup of (near) head-on collisions, suppresses the radiative process, while the Doppler-shifted (effectively unipolar) fields seen by the electron on its trajectory enhance the nonradiative processes. Our results thus motivate the study of (near) tail-on collisions of electrons with intense laser pulses, in order to study purely quantum spin effects in intense laser fields.

Our investigation has also underlined several results which have a bearing on other investigations of electron spin in strong fields. First, in plane waves, there can be no spin flip without loops or emissions. As such the leadingorder BMT equation commonly studied in the literature describes only the precession of the classical spin vector needed to account for the acceleration of the electron by the field; it does not describe any change in the spin state of the electron. Second, because of this result, there is also no order $\alpha^{1}$ nonradiative spin flip in a plane wave, and so any attempt to infer from plane wave results (e.g., via the LCFA) for more general, focused beams, fail outright. (This is in contrast to radiative spin flip through nonlinear Compton.) As we saw above, predictions which neglect the order $\alpha$ interference terms are incorrect.

Third, in focused laser pulses, focusing effects dramatically alter the dynamics of nonradiative spin flip compared to the plane wave case. The sensitivity of spin flip to focusing effects in the background can be greater than that to loop effects, meaning that focused fields must be modeled to very high precision in order to correctly report spin-flip effects where nonradiative processes play a significant role. (This is at low energy, since NLC dominates spin flips at higher energies.) This would suggest that extreme care is needed, in simulations and experimental modeling, in parameter regions where BMT contributes an appreciable amount to the overall probability for flip of the electron spin.

\section{ACKNOWLEDGMENTS}

The authors are supported by the EPSRC, Grant No. EP/ S010319/1.

\section{APPENDIX: SPIN BASIS}

Let $\sigma_{i}$ be the $2 \times 2$ Pauli matrices. In the Kogut-Soper basis for the $\gamma$-matrices [41],

$$
\begin{array}{ll}
\gamma^{0}=\left[\begin{array}{ll}
0 & \mathbb{1} \\
\mathbb{1} & 0
\end{array}\right], \quad \gamma^{1}=\left[\begin{array}{cc}
0 & -\sigma_{1} \\
\sigma_{1} & 0
\end{array}\right], \\
\gamma^{2}=\left[\begin{array}{cc}
0 & -\sigma_{2} \\
\sigma_{2} & 0
\end{array}\right], \quad \gamma^{3}=\left[\begin{array}{cc}
0 & -\sigma_{3} \\
\sigma_{3} & 0
\end{array}\right],
\end{array}
$$

and $\gamma^{5}=i \gamma^{0} \gamma^{1} \gamma^{2} \gamma^{3}$, the explicit form of the lightfront helicity spinors $u_{p \sigma}$ in our discussion is

$$
\begin{gathered}
u_{p+}=\frac{1}{\sqrt{2 m p^{-}}}\left[\begin{array}{c}
p^{1}-i p^{2} \\
p^{-} \\
0 \\
m
\end{array}\right], \\
u_{p-}=\frac{1}{\sqrt{2 m p^{-}}}\left[\begin{array}{c}
m \\
0 \\
p^{-} \\
-p^{1}-i p^{2}
\end{array}\right] .
\end{gathered}
$$

In Cartesian components, the basis elements used in the BMT calculation, as defined in and below (22), obey

$$
\begin{aligned}
& S_{++}^{\mu}=-S_{--}^{\mu}=h_{\pi}^{\mu}, \\
& S_{+-}^{\mu}=\left(\frac{\pi^{1}+i \pi^{2}}{\pi^{-}}, 1, i, \frac{\pi^{1}+i \pi^{2}}{\pi^{-}}\right), \\
& S_{-+}^{\mu}=S_{+-}^{\mu *} .
\end{aligned}
$$

where $n \cdot S_{++}=\pi^{-} / m$ and $n \cdot S_{+-}=0$. Note that $\pi^{\mu}$ here stands for the in-principle time-dependent momentum of the state.
[1] J. M. Cole et al., Phys. Rev. X 8, 011020 (2018).

[2] K. Poder et al., Phys. Rev. X 8, 031004 (2018).

[3] N. V. Elkina, A. M. Fedotov, I. Y. Kostyukov, M. V. Legkov, N. B. Narozhny, E. N. Nerush, and H. Ruhl, Phys. Rev. ST Accel. Beams 14, 054401 (2011).

[4] C. Ridgers, J. G. Kirk, R. Duclous, T. Blackburn, C. Brady, K. Bennett, T. Arber, and A. Bell, J. Comput. Phys. 260, 273 (2014).
[5] A. Gonoskov, S. Bastrakov, E. Efimenko, A. Ilderton, M. Marklund, I. Meyerov, A. Muraviev, A. Sergeev, I. Surmin, and E. Wallin, Phys. Rev. E 92, 023305 (2015).

[6] A. I. Nikishov and V. I. Ritus, Sov. Phys. JETP 19, 529 (1964).

[7] L. S. Brown and T. W. B. Kibble, Phys. Rev. 133, A705 (1964).

[8] V. I. Ritus, J. Russ. Laser Res. 6, 497 (1985). 
[9] B. King, Phys. Rev. A 91, 033415 (2015).

[10] D. Seipt and B. King, arXiv:2007.11837 [Phys. Rev. A (to be published)].

[11] M. Boca and V. Florescu, Phys. Rev. A 80, 053403 (2009).

[12] K. Krajewska and J. Kaminski, Laser Part. Beams 31, 503 (2013).

[13] K. Krajewska and J. Z. Kamiński, Phys. Rev. A 90, 052117 (2014).

[14] M. J. A. Jansen, J. Z. Kamiński, K. Krajewska, and C. Müller, Phys. Rev. D 94, 013010 (2016).

[15] T. N. Wistisen and A. Di Piazza, Phys. Rev. D 100, 116001 (2019).

[16] D. Seipt, D. Del Sorbo, C. Ridgers, and A. R. Thomas, Phys. Rev. A 98, 023417 (2018).

[17] D. Del Sorbo, D. Seipt, T. G. Blackburn, A. G. R. Thomas, C. D. Murphy, J. G. Kirk, and C. P. Ridgers, Phys. Rev. A 96, 043407 (2017).

[18] D. D. Sorbo, D. Seipt, A. G. R. Thomas, and C. P. Ridgers, Plasma Phys. Controlled Fusion 60, 064003 (2018).

[19] D. Seipt, D. Del Sorbo, C. P. Ridgers, and A. G. R. Thomas, Phys. Rev. A 100, 061402 (2019).

[20] V. N. Bă̌er, V. M. Katkov, A. I. Mil'shteĭn, and V. M. Strakhovenko, Sov. Phys. JETP 42, 400 (1976).

[21] S. Meuren and A. Di Piazza, Phys. Rev. Lett. 107, 260401 (2011).

[22] M. Lavelle and D. McMullan, Phys. Lett. B 798, 135021 (2019).

[23] V. Ritus, Sov. Phys. JETP 30, 1113 (1970).

[24] N. Narozhnyi, Phys. Rev. D 20, 1313 (1979).

[25] N. Narozhnyi, Phys. Rev. D 21, 1176 (1980).

[26] A. Fedotov, J. Phys. Conf. Ser. 826, 012027 (2017).

[27] T. Podszus and A. Di Piazza, Phys. Rev. D 99, 076004 (2019).

[28] A. Ilderton, Phys. Rev. D 99, 085002 (2019).

[29] A. Ilderton, Phys. Rev. D 100, 125018 (2019).

[30] A. Mironov, S. Meuren, and A. Fedotov, Phys. Rev. D 102, 053005 (2020).

[31] V. Yakimenko et al., Phys. Rev. Lett. 122, 190404 (2019).

[32] C. Baumann, E. Nerush, A. Pukhov, and I. Kostyukov, Sci. Rep. 9, 9407 (2019).

[33] T. Blackburn, A. Ilderton, M. Marklund, and C. Ridgers, New J. Phys. 21, 053040 (2019).

[34] A. Di Piazza, T. Wistisen, M. Tamburini, and U. Uggerhøj, Phys. Rev. Lett. 124, 044801 (2020).

[35] Y.-F. Li, R. Shaisultanov, K. Z. Hatsagortsyan, F. Wan, C. H. Keitel, and J.-X. Li, Phys. Rev. Lett. 122, 154801 (2019).
[36] F. Wan, R. Shaisultanov, Y.-F. Li, K. Z. Hatsagortsyan, C. H. Keitel, and J.-X. Li, Phys. Lett. B 800, 135120 (2020).

[37] J. Thomas, A. Hützen, A. Lehrach, A. Pukhov, L. Ji, Y. Wu, X. Geng, and M. Büscher, Phys. Rev. Accel. Beams 23, 064401 (2020).

[38] V. N. Baier, Sov. Phys. Usp. 14, 695 (1972).

[39] E. Leader and C. Lorcé, Phys. Rep. 541, 163 (2014).

[40] P. A. Dirac, Rev. Mod. Phys. 21, 392 (1949).

[41] S. J. Brodsky, H.-C. Pauli, and S. S. Pinsky, Phys. Rep. 301, 299 (1998).

[42] T. Heinzl, Lect. Notes Phys. 572, 55 (2001).

[43] B. Bakker et al., Nucl. Phys. B, Proc. Suppl. 251-252, 165 (2014).

[44] V. Dinu, T. Heinzl, and A. Ilderton, Phys. Rev. D 86, 085037 (2012).

[45] V. Bargmann, L. Michel, and V. L. Telegdi, Phys. Rev. Lett. 2, 435 (1959).

[46] M. Jacob and G. Wick, Ann. Phys. (N.Y.) 7, 404 (1959).

[47] K. Y.-J. Chiu and S. J. Brodsky, Phys. Rev. D 95, 065035 (2017).

[48] C. Itzykson and J. Zuber, Quantum Field Theory (McGrawHill, New York, 1980).

[49] D. M. Volkov, Z. Phys. 94, 250 (1935).

[50] I. A. Aleksandrov, D. A. Tumakov, A. Kudlis, V. M. Shabaev, and N. N. Rosanov, Phys. Rev. A 102, 023102 (2020).

[51] V. Dinu, Phys. Rev. A 87, 052101 (2013).

[52] D. Seipt, arXiv:1701.03692.

[53] A. Ilderton and G. Torgrimsson, Phys. Rev. D 87, 085040 (2013).

[54] L. Bieri and D. Garfinkle, Classical Quantum Gravity 30, 195009 (2013).

[55] A. Di Piazza, M. Tamburini, S. Meuren, and C. H. Keitel, Phys. Rev. A 99, 022125 (2019).

[56] A. Ilderton, B. King, and D. Seipt, Phys. Rev. A 99, 042121 (2019).

[57] F. W. Olver, D. W. Lozier, R. F. Boisvert, and C. W. Clark, NIST Handbook of Mathematical Functions (Cambridge University Press, Cambridge, England, 2010).

[58] C. N. Harvey, A. Ilderton, and B. King, Phys. Rev. A 91, 013822 (2015).

[59] A. Di Piazza, M. Tamburini, S. Meuren, and C. H. Keitel, Phys. Rev. A 98, 012134 (2018).

[60] T. Heinzl, B. King, and A. MacLeod, arXiv:2004.13035.

[61] Y. I. Salamin and C. H. Keitel, Phys. Rev. Lett. 88, 095005 (2002).

[62] B. King and H. Hu, Phys. Rev. D 94, 125010 (2016). 